\title{
Impact of Mobility Models on Social Structure Formation in Opportunistic Networks
}

\author{
Halikul Lenando*, Mohamad Nazim Jambli**, Kartinah Zen***, Johari Abdullah***** \\ Faculty Computer Science and Information technology \\ Universiti Malaysia Sarawak \\ Kota Samarahan, Kuching Sarawak \\ Email:cool@fit.unimas.my*,jmnazim@fit.unimas.my**,kartinah@qad.unimas.my***,ajohari@fit.unimas.my****
}

\begin{abstract}
In mobile opportunistic networks, the network topology is unpredictable and very dynamic due to the existence of the mobile nodes in the network. The nodes' mobility affect the nodes' interaction frequency. Hence, it also affecting the social structure formation between nodes. In this paper, we study the impact of different mobility models namely Random Walk, Random Waypoint, Gauss Markov and D-GM on the social structure formation in opportunistic networks. The study shows that different mobility models have different impact on the social structures formation. Based on our experimental results, the Gauss Markov model provides better social structure compared to others because it creates more opportunities for a node to interact with different nodes.
\end{abstract}

Index Terms-Social Networks ;Mobility Model ; Opportunistic networks;

\section{INTRODUCTION}

An opportunistic network has been developed by the networking community as an evolution of delay tolerant networking. Instead of end-to-end connectivity being available at a single point in time, connections may be intermittent and so the path of links between source and recipient is spread over a period of time, maybe with no complete path ever existing between sender and receiver [1]. The frequency of interactions between mobile devices is dependent on the mobile devices' mobility. Furthermore, mobility model plays a very important role in data dissemination in opportunistic network. Hence, it is vital to study and analyze the impact of different mobility models on the formation social structure.

In opportunistic networks, mobility model acts as a routing protocol. Routing protocol determines how data should be forwarded from one node to another nodes. Numbers of studies have been conducted to understand the impact of mobility model in the networks that has dynamic topology such as Mobile Ad-Hoc Networks (MANETs) [2], Mobile sensor Network [3] and opportunistic networks [4].

The impact of mobility model on MANET routing protocol has been studied in [5]. In this work the difference routing protocols are compared in terms of their performance using different mobility model. Similar in [2], different mobility models are used to compare the performance of two protocols in mobile sensor network. In this paper, we compare the impact of different mobility model on social structure formation using sliding window social structure approach [4]. This work is part of research project on opportunistic networks to investigate the performance of data dissemination in mobile environment.

\section{MobiLity MODEL}

Nodes in the simulation model move based on the Random Walk, Random Waypoint and Gauss Markov mobility models. Random Walk and Random Waypoint are categorized as movement independent, which means next movement is not influenced by its previous attributes. In contrast with Gauss Markov next location of a node is calculated based on the previous speed and direction. The following subsections describe the four mobility models used in the simulation.

1) Random Walk: Initially, each mobile node is given two random parameters, direction and speed. The node travels along the trajectory for a fix time interval. In our simulation, we assumed that nodes changed direction and speed every 30 seconds as to mimic the randomness of the human who walks randomly in open space. We called this time value an interval. For each interval, a new direction and speed will be assigned randomly. Note that, using a very short time interval, will confine the node movement in a small area because there is a possibility of nodes to move to their original position after moving to different locations.

2) Random Waypoint: Random waypoint is an extension of Random walk. This model has a pause time at each interval time, where nodes have to stay at a location for a certain period of time (pause). Before the node moves to a new location, the will be using a new direction (generated randomly) and speed which uniformly distributed between [minSpeed, maxSpeed] . In our simulation, the speed range is chosen between $[4 \mathrm{~km} / \mathrm{h}$, $8 \mathrm{~km} / \mathrm{h}$ ] which suite the pedestrians' walking speed. The pause time at each destination is 30 seconds.

3) Gauss Markov: Initially, each mobile node is assigned a random speed and direction. At fix interval time $n$, new value of speed and direction is calculated based on the following formula [6].

$$
\begin{aligned}
& s_{n}=\alpha s_{n-1}+(1-\alpha) \bar{s}+\sqrt{\left(1-\alpha^{2}\right)} s_{x_{n-1}} \\
& d_{n}=\alpha d_{n-1}+(1-\alpha) \bar{d}+\sqrt{\left(1-\alpha^{2}\right)} d_{x_{n-1}}
\end{aligned}
$$

Here $s_{n}, d_{n}$ is the new speed and direction at time interval n. $\alpha$,where $0 \leq \alpha \leq 1$, is the tuning parameter used to vary 